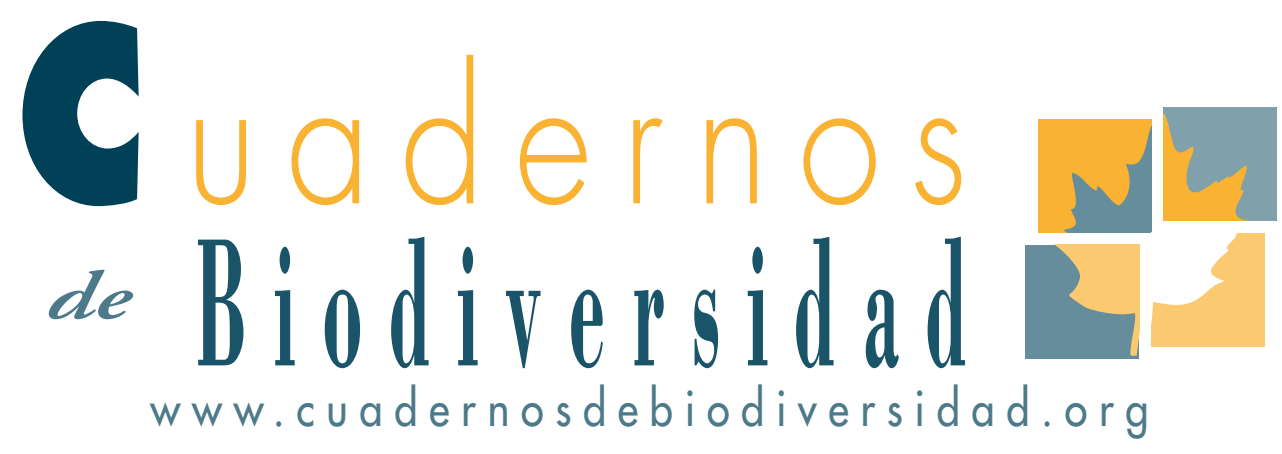

\title{
Migración e invernada del Halcón de Eleonora (Falco eleonorae Gené, 1839; falconidae). ¿Qué hemos aprendido con el seguimiento por satélite?
}

\section{Rubén Limiñana*, **, Ugo Mellone ${ }^{* *}$, Pascual López-López ${ }^{* *}$ \& Vicente Urios ${ }^{* *}$}

* Instituto de Investigación en Recursos Cinegéticos (IREC). CSIC-UClM-JCCM. Ronda de Toledo, s/N. i307i Ciudad Real (España).

** Estación Biológica Terra Natura. Grupo de Investigación Zoología de Vertebrados. Instituto Universitario de Investigación CibiO. Universidad de Alicante. Campus San Vicente del Raspeig, s/N. 03080 Alicante (España).

\section{ABSTRACT}

In recent years, satellite tracking has enabled to get new important insights into several aspects of bird's migration, one of the most unknown aspects of birds' ecology. In the last years, we have studied the migration and wintering of satellite tracked Eleonora's falcons (Falco eleonorae Gené, 1839) tagged during the breeding season at Balearic and Columbretes Islands. In autumn, Eleonora’s falcons migrated following inland routes along Africa. Birds arrived into Africa flying over the Mediterranean Sea directly from the breeding colonies, and then crossed the Sahara Desert, the Sahel region, south-eastern African savannahs and the Channel of Mozambique to arrive into their wintering grounds in Madagascar.
During winter, falcons used areas of pristine humid forests close to degraded forests and cultivations, where they can fulfil food requirements for this period. In spring, falcons travelled larger over-sea distances between Madagascar and continental Africa than in autumn. We observed inter-annual variations in the routes used in this season to cross to inland Africa, which were related to meteorological conditions at the time of leaving Madagascar. These results are very important to propose global conservation actions for the species, highlighting that these actions should embrace the whole lifecycle of the species. 


\section{KEY WORDS:}

Conservation, Eleonora's falcon, migration, raptors, satellite tracking, wintering.

\section{INTRODUCCIÓN}

El halcón de Eleonora (Falco eleonorae Gené, 1839) es una de las especies de ave de presa nidificante en España más desconocida y que mayor interés por su conservación ha despertado en los últimos años. Esta especie de halcón nidifica principalmente en islas del Mediterráneo (aunque también presenta colonias de cría en las Islas Canarias y costa atlántica de Marruecos). Se trata de una de las pocas especies de rapaces que nidifican en islas y cuyas poblaciones son íntegramente migratorias (Ferrer et al., 2011). Al contrario que el resto de aves migratorias que nidifican en Europa, las cuales tienen su periodo de nidificación entre abril y julio, el halcón de Eleonora presenta un periodo de nidificación retrasado, que se inicia en julio y termina entre finales de septiembre y principio de octubre. De este modo, los halcones de Eleonora son capaces de aprovechar las oleadas de paseriformes migrantes que usan las islas como lugares de parada, haciendo coincidir la disponibilidad temporal y puntual de este recurso trófico con el periodo de cría de los juveniles, siendo esta una etapa en la que es necesario un gran aporte de comida.

La principal zona de invernada de la especie, conocida mediante las recuperaciones de anillas de aves y observaciones de campo, es la isla de Madagascar (Walter, 1979; Ferguson-Lees \& Christie, 2001). Al tratarse de una especie que nidifica en ambientes marinos, para alcanzar Madagascar desde las islas del Mediterráneo, se pensaba que los halcones de Eleonora seguían rutas migratorias sobre el mar y las costas, cruzando primero hacia el Mar Rojo a través del Canal de Suez y bordeando luego la costa este de África hasta llegar a Madagascar (Stresemann, 1954; Walter, 1979). Sin embargo, hasta fechas muy recientes esta ruta era sólo una hipótesis, al no ser posible seguir individualmente a los halcones de
Eleonora durante sus migraciones. Los únicos estudios existentes se basaban en la recopilación de datos de anillamiento y observaciones en algunos lugares puntuales de las rutas migratorias de la especie (Delgado \& Quilis, 1990), los cuales proporcionan una información restringida sobre la migración.

En los últimos ańos, la telemetría satelital se ha convertido en una herramienta muy importante y útil para el estudio de los movimientos de las aves (ver Sokolov, 2011). En particular, en el caso de las aves migratorias, este método permite estudiar las estrategias de migración, tanto a nivel individual como poblacional, además de servir para obtener información sobre las especies seguidas en regiones muy lejanas a sus zonas de nidificación (Jiguet et al., 2011; Limiñana et al., 2012). Esto permite tener información, al menos de manera indirecta, sobre los problemas de conservación de estas aves en regiones alejadas de los lugares de cría, siendo en estos últimos donde se realizan prácticamente todas las acciones encaminadas a la conservación, pudiendo resultar ineficaces si no se considera todo el ciclo anual (Martin et al., 2007).

\section{METOdOLOGÍA}

Entre los años 2007 y 2010 marcamos un total de 17 halcones de Eleonora con emisores satelitales de las colonias de cría de Islas Columbretes e Islas Baleares (Tagomago, Isla de Dragonera y Mallorca), con el objetivo de estudiar las rutas de migración y zonas de invernada de la especie. Los ejemplares fueron capturados mediante el uso de redes japonesas modificadas para la captura de rapaces, usando como seńuelo para atraer a los halcones un búho real (Bubo bubo) disecado. Se usaron emisores satelitales de tipo Argos (http://www.argos-system.org) de 9.5 gramos de peso, que fueron colocados en el dorso de las aves mediante un arnés de teflón, que es un material resistente a la vez que poco abrasivo y por tanto, no perjudicial para las aves (Figura 1). Los ejemplares fueron liberados en un máximo de 30 minutos tras ser capturados. 


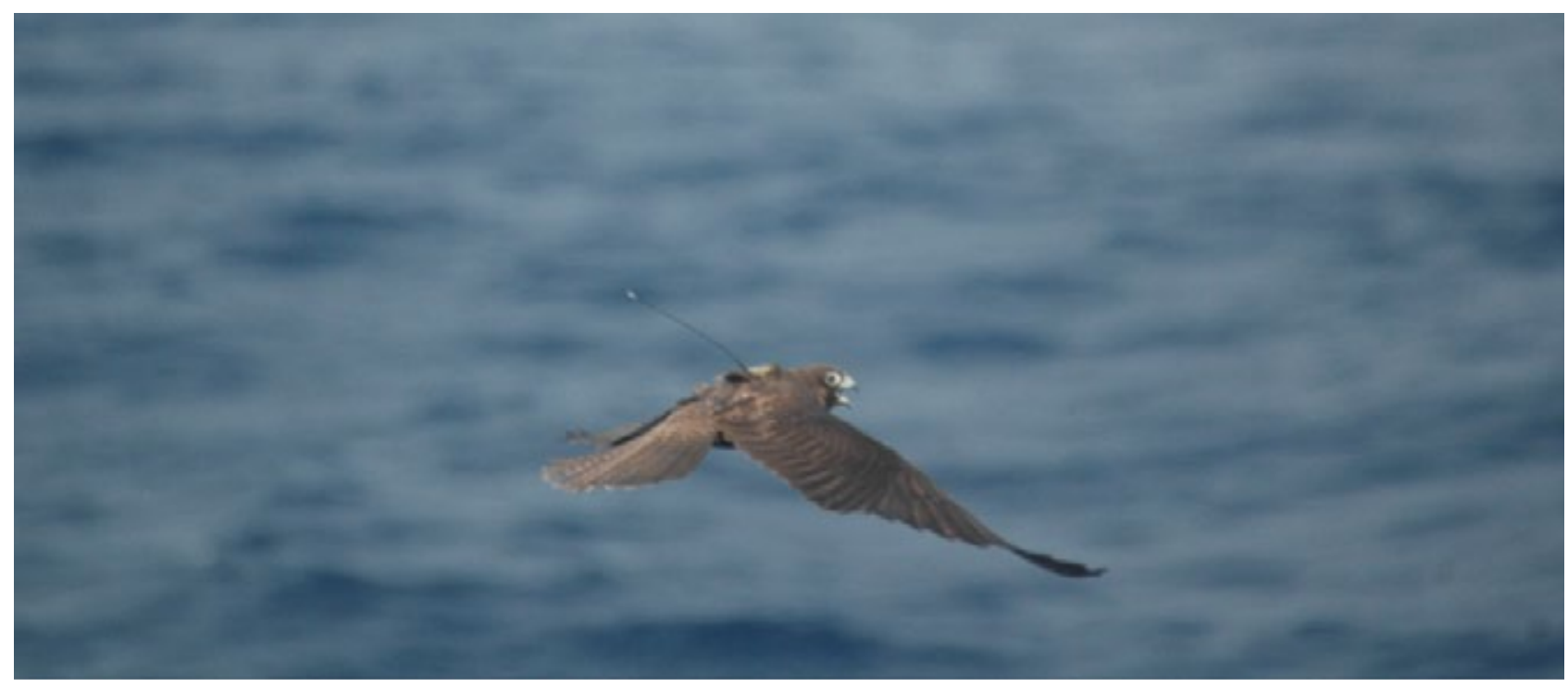

Figura 1. Ejemplar adulto de halcón de Eleonora (Falco eleonorae) marcado con un dispositivo de seguimiento vía satélite (Foto: Vicente Urios).

\section{RUTAS DE MIGRACIÓN OTOÑAL}

Lo primero que se descubrió mediante estos marcajes es que los halcones de Eleonora no usan rutas de migración marinas, como se creía hasta ese momento, sino que cruzan hasta el norte de África directamente desde las colonias de nidificación (los ejemplares marcados llegaron principalmente a Argelia), cruzando luego el Desierto del Sahara, la región del Sahel, bosques tropicales y ecuatoriales y por último el Canal de Mozambique hasta alcanzar Madagascar (López-López et al., 2009, 2010). Durante toda esta migración, todos los ejemplares siguen unas rutas en forma de S-invertida, recorriendo casi 10.000 kilómetros entre sus zonas de nidificación e invernada (Figura 2). Ejemplares de la especie marcados en Grecia e Italia muestran rutas de migración similares a los ejemplares seguidos desde España (Gschweng et al., 2008; Kassara et al., 2012).

El vuelo sobre el Desierto del Sahara, considerado una barrera ecológica ya que las posibilidades de alimentación son muy escasas por su extrema aridez, implica un gasto energético muy grande para estas aves, más aún teniendo en cuenta el resto del viaje que todavía les queda por completar. Para cruzar el desierto lo más rápido posible, los halcones vuelan tanto de día como de noche cuando sobrevue- lan esta barrera ecológica, alcanzando así velocidades diarias más altas que en otras regiones (López-López et al., 2010). Sin embargo, después de cruzar el desierto, los halcones llegan a las llanuras del Sahel, donde aprovechan estos ambientes más productivos para cazar y comer, como sugieren las velocidades más bajas, la menor proporción de tiempo volando de noche y las rutas más sinuosas que se observan en esta región (López-López et al., 2010). Las aves cruzan después los bosques tropicales y ecuatoriales del centro de África, llegando así al lago Victoria. Desde esta importante referencia geográfica, sólo les queda cruzar las sabanas de Tanzania para llegar a la última barrera geográfica que los separa de los cuarteles de invernada: el canal de Mozambique. El cruce de este canal implica un vuelo de más de 600 kilómetros sobre el mar, sin posibilidad de paradas, por lo que los halcones cruzaron este brazo de mar sin detenerse y volando tanto de día como de noche, alcanzando también altas velocidades (López-López et al., 2010). Pese a tratarse de un largo cruce de mar, los halcones seleccionan para cruzar las rutas más cortas entre la costa africana y Madagascar (Figura 2). De manera general, durante esta migración otoñal, el halcón de Eleonora recorre distancias medias de unos 200-300 kilómetros al día, alcanzando velocidades máximas de hasta 70-80 kilómetros/hora. 


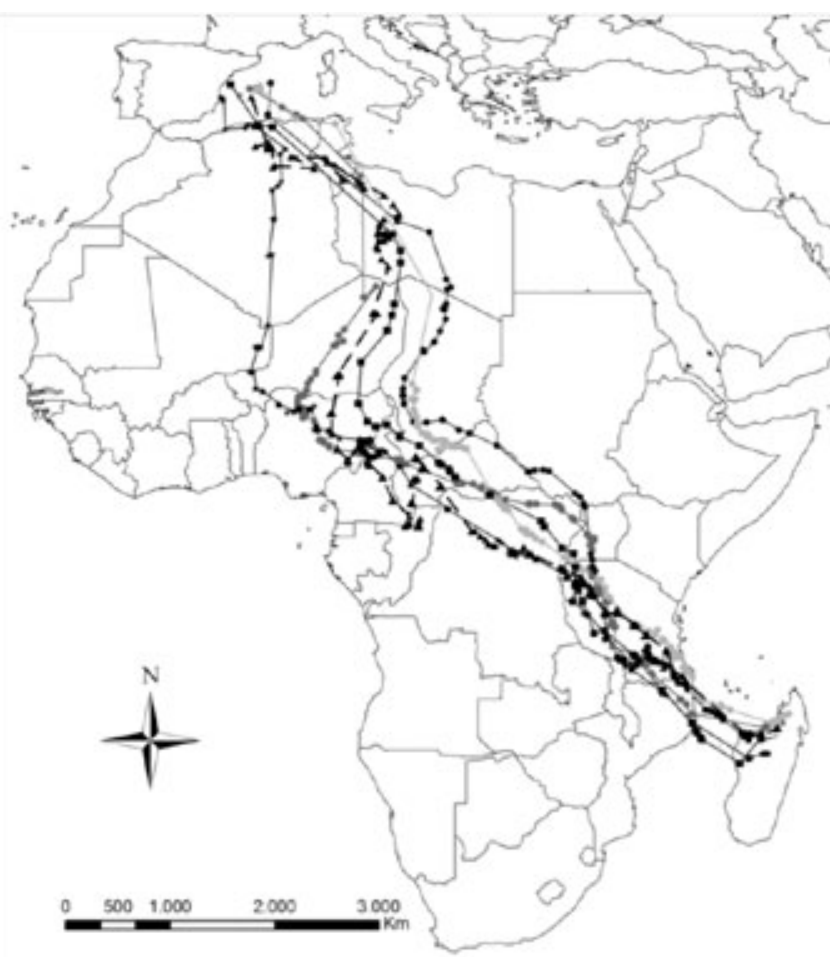

Figura 2. La telemetría satelital ha revelado algunos aspectos muy interesantes de la ecología del halcón de Eleonora (Falco eleonorae). Por ejemplo, véase las rutas de migración otoñales de la especie.

\section{RUTAS DE MIGRACIÓN PRIMAVERAL}

Durante la migración primaveral de vuelta a las zonas de cría, los halcones de Eleonora deben deshacer todo el camino realizado el otoño anterior, lo que implica recorrer de nuevo los casi 10.000 kilómetros hasta las zonas de nidificación en el Mediterráneo. Sin embargo, los resultados más interesantes de toda esta ruta primaveral se han obtenido durante el cruce del Canal de Mozambique, entre Madagascar y la costa oriental de África. En el año 2009, tres halcones de Eleonora volaron desde el norte de Madagascar hasta las costas de Somalia, realizando un vuelo sobre el mar (y por lo tanto sin paradas), de aproximadamente 1.500 kilómetros de distancia. Al año siguiente, dos de esos halcones siguieron una ruta distinta, esta vez llegando hasta las costas de Tanzania, volando sobre el mar unos 1.200 kilómetros. Esto supone unos de los vuelos sin paradas sobre el mar más largos registrados para aves rapaces. Estas diferencias en el cruce entre
África y Madagascar observadas entre años diferentes se deben a diferentes condiciones meteorológicas (Mellone et al., 2011). En el 2009, al inicio de la migración, se observó la existencia de borrascas sobre el Canal de Mozambique, asociada a fuertes vientos y malas condiciones atmosféricas en general, lo que dificultó por tanto el vuelo, por lo que los halcones usaron rutas más largas pero más seguras. Esto les permite aumentar las posibilidades de completar este cruce de mar de manera satisfactoria. Por el contrario, la situación fue diferente en 2010, donde se dieron condiciones meteorológicas más favorables en el canal de Mozambique. Ese año los halcones utilizaron una vía más corta y directa en su cruce de retorno a África continental. Gracias a la telemetría vía satélite se ha comprobado que también existen barreras meteorológicas que afectan y definen las rutas de migración de las aves y que las aves tienen una cierta plasticidad para adaptarse a las condiciones experimentadas durante las migraciones, con el fin de poder completar con éxito sus largos viajes.

\section{USO DEL HÁBITAT DURANTE LA INVER- NADA}

Como se ha comentado anteriormente, los halcones de Eleonora pasan la época reproductora en ambientes marinos. Sin embargo, durante la invernada, usan hábitats totalmente distintos. En Madagascar, los halcones de Eleonora se encuentran en zonas de bosques húmedos prístinos (de acuerdo con los tipos de hábitats definidos en el Madagascar Vegetation Mapping Project, http://www.vegmad. org), los cuales se encuentran situados en áreas situadas sobre los 1.200 metros de altitud, especialmente en el nordeste de de la isla (Mellone et al., 2012a; Kassara et al., 2012; Gschweng et al., 2012). Lo más interesante es que seleccionan positivamente zonas de bosques degradados o zonas de cultivos próximos a estos bosques húmedos (Mellone et al., 2012a). De este modo, los halcones de Eleonora se aprovechan de la mayor cantidad de presas (fundamentalmente insectos) que aparecen en estas zonas de ecotono, siendo además más fáciles de capturar en zonas más abiertas que dentro de los propios bosques. Sin embargo, los bosques en sí son muy importantes, ya 
que son el tipo de hábitat más abundante dentro de las áreas de campeo de los ejemplares seguidos en Madagascar (Mellone et al., 2012a). Por lo tanto, lo que seleccionan estos halcones durante la invernada son bosques bien conservados, donde seguramente se desarrollan muchos de los insectos de los que se alimentan, cerca de otros tipos de bosques más degradados o zonas de cultivos, donde es más fácil cazarlos. Estos bosques húmedos son uno de los hábitats más amenazados de la isla y están siendo transformados en la actualidad, lo que pone en peligro la conservación del halcón de Eleonora, así como de otras especies residentes y migratorias.

\section{CONCLUSIONES Y PERSPECTIVAS}

El halcón de Eleonora es una especie cuyo ciclo anual tiene lugar en una gran cantidad de hábitats, desde islas del Mediterráneo hasta bosques húmedos de Madagascar, pasando por desiertos y llanuras tropicales, separados entre sí miles de kilómetros de distancia. Esto implica que las amenazas que pueda sufrir a lo largo del año son muy variadas y dependen de la región del planeta en la que se encuentren. Por citar algunas amenazas, en las zonas de cría se han detectado intoxicaciones cuando los halcones acuden a beber agua dulce a pequeñas charcas (Ristow, 2001). También pueden verse amenazados por el impacto del elevado número de turistas en algunas islas (Martínez-Abraín et al., 2002). Por el contrario, la pérdida de hábitat en Madagascar parece ser la principal amenaza durante la época no reproductora.

Además de lo anteriormente expuesto, cruzar el desierto del Sáhara supone uno de los mayores retos a los que se enfrentan las aves migratorias debido al elevado riesgo de mortalidad asociado (Strandberg et al., 2010). También, la degradación de las condiciones ambientales en las zonas por las que transcurren las rutas de migración, pueden tener un impacto negativo en estas aves (Newton, 2008), ya que puede disminuir las posibilidades de supervivencia durante las migraciones, lo que tendría un efecto directo en el número de ejemplares que iniciarán la siguiente temporada de cría y, por lo tanto, en la dinámica poblacional. Finalmente, nuestros estudios demuestran que las condiciones ambientales tienen una gran influencia en las rutas y el efecto del viento puede hacer que las aves puedan ser desplazadas hacia ambientes más severos que pueden hacer disminuir la probabilidad de completar las migraciones con éxito (Mellone et al., 2012b).

Como hemos comprobado, la telemetría satelital proporciona una valiosa información sobre la ecología de las aves migratorias, lo cual puede ser empleado para proponer estrategias adecuadas para la conservación de estas especies, las cuales deben considerar todo el ciclo anual y no sólo una parte del mismo (Martin et al., 2007). Poder obtener mayor número de datos con este tipo de dispositivos constituye un gran reto. Por otro lado, la combinación de este conocimiento con los datos de seguimiento de campo de las especies (e.g. reproducción, comportamiento,...), proporciona una información muy valiosa sobre su ecología, al poder relacionar directamente los eventos de nidificación en Europa, con los que ocurren durante las migraciones o la invernada, a varios miles de kilómetros.

\section{BIBLIOGRAFÍA}

Delgado, G. \& Quilis, V. 1990. New data concerning the migration of Eleonora's Falcon Falco eleonorae. Ringing Migrat. 11:111-112.

Ferguson-Lees, J. \& Christie, D.A. 2001. Raptors: birds of prey of the world. A \& C Black Publishers. London. 992 pp.

Ferrer, M., Bildstein, K., Penteriani, V., Casado, E. \& de Lucas, M. 2011. Why birds with deferred sexual maturity are sedentary on islands: a systematic review. PLOS ONE 6(7):e22056.

Gschweng, M., Kalko, E.K.V., Querner, U., Fiedler, W. \& Berthold, P. 2008. All across Africa: highly individual migration routes of Eleonora's falcon. Proc. R. Soc. Lond. B. $275: 2887-2896$.

Gschweng, M., Kalko, E.K.V., Berthold, P., Fiedler, W \& Fahr, J. 2012. Multi-temporal distribution modelling with satellite tracking data: predicting responses of a longdistance migrant to changing environmental conditions. J. Appl. Ecol. DOI: 10.1111/j.1365-2664.2012.02170.x 
Jiguet, F., Barbet-Massin, M. \& Chevalier, D. 2011. Predictive distribution models applied to satellite tracks: modelling the western African winter range of European migrant Black Storks Ciconia nigra. J. Ornithol. 152: $111-118$.

Kassara, C., Fric, J., Gschweng, M. \& Sfenthourakis, S. 2012. Complementing the puzzle of Eleonora's Falcon (Falco eleonorae) migration: new evidence from an eastern colony in the Aegean Sea. J. Ornithol. 153:839-848.

Limiñana, R., Soutullo, A., Arroyo, B. \& Urios, V. 2012. Protected areas do not fulfil the wintering habitat needs of the trans-Saharan migratory Montagu's harrier. Biol. Conserv. 145:62-69.

López-López, P., Limiñana, R. \& Urios, V. 2009. Autumn migration of Eleonora's falcon Falco eleonorae tracked by satellite telemetry. Zool. Stud. 48:485-491.

López-López, P., Limiñana, R., Mellone, U. \& Urios, V. 2010. From the Mediterranean Sea to Madagascar. Are there ecological barriers from the long-distance migrant Eleonora's falcon? Landscape Ecol. 25:803-813.

Martin, T. G., Chadès, I., Arcese, P., Marra, P.P., Possingham, H.P. \& Norris, D.R. 2007. Optimal conservation of migratory species. PLoS ONE 2(8):e751.

Martínez-Abraín, A., Oro, D., Ferris, V. \& Belenguer, R. 2002. Is growing tourist activity affecting the distribution or number of breeding pairs in a small colony of the Eleonora's Falcon? Anim. Biodiver. Conserv. 25:47-51.

Mellone, U., López-López, P., Limiñana, R. \& Urios, V. 2011. Weather conditions promote route flexibility during open ocean crossing in a long-distance migratory raptor. Int. J. Biometeorol. 55:463-468.
Mellone, U., López-López, P., Limiñana, R. \& Urios, V. 2012a. Wintering habitats of Eleonora's falcons Falco eleonorae in Madagascar. Bird Study 59:29-36.

Mellone, U., Klaassen, R.H.G., García-Ripollés, C., Limiñana, R., López-López, P., Pavón, D., Strandberg, R., Urios, V., Vardakis, M. \& Alerstam, T. 2012b. Interspecific Comparison of the Performance of Soaring Migrants in Relation to Morphology, Meteorological Conditions and Migration Strategies. PLoS ONE 7(7):e39833.

Newton, I. 2008. The Migration Ecology of Birds. Academic Press. London. 976 pp.

Ristow, D. 2001. Poison is causing the sudden population decline of Eleonora's falcon. International Hawkwatcher 3:10-17.

Sokolov, L.V. 2011. Modern Telemetry: New Possibilities in Ornithology. Biol. Bull. 38:885-904.

Strandberg, R., Klaassen, R.H.G., Hake, M. \& Alerstam, T. 2010. How hazardous is the Sahara Desert crossing for migratory birds? Indications from satellite tracking of raptors Biol. Lett. 6:297-300.

Stresemann, E. 1954. Zur Frage der Wanderungen des Eleonorenfalken. Vogelwarte 17:182-183.

Walter, H. 1979. Eleonora's Falcon. Adaptations to Prey and Habitat in a Social Raptor. The University of Chicago Press. London. 